\title{
Genetic strategy for analyzing specificity of dimer formation: Escherichia coli cyclic AMP receptor protein mutant altered in its dimerization specificity
}

\author{
J. Keith Joung, Eugene H. Chung, Gareth King, Channing Yu, Andrew S. Hirsh, \\ and Ann Hochschild ${ }^{1}$ \\ Department of Microbiology and Molecular Genetics, Harvard Medical School, Boston, Massachusetts 02115 USA
}

\begin{abstract}
Many transcriptional regulators function in homo- or heterodimeric combinations. The same protein can carry out distinct regulatory functions depending on the partner with which it associates. Here, we describe a mutant of the Escherichia coli cAMP receptor protein (CRP) that has an altered dimerization specificity; that is, mutant/mutant homodimers form preferentially over wild-type/mutant heterodimers. CRP dimerization involves the formation of a parallel coiled-coil structure, and our CRP mutant bears an amino acid substitution affecting the first " $d$ " position residue within the $\alpha$-helix that mediates CRP dimerization. The genetic strategy we used to isolate this CRP altered dimerization specificity (ADS) mutant is generalizable and could be utilized to isolate ADS mutants of other dimeric transcriptional regulators.
\end{abstract}

[Key Words: CRP; dimerization specificity; heterodimers; coiled coil; transcriptional regulators; genetics]

Received May 18, 1995; revised version accepted October 13, 1995.

Many transcriptional regulators in both prokaryotes and eukaryotes bind DNA as dimers. Several structural motifs have been described that mediate dimerization (e.g., the leucine zipper and the helix-loop-helix motifs) (Landschultz et al. 1988; O'Shea et al. 1991; Ferré-D'Amaré et al. 1993; Ellenberger et al. 1994), and families of structurally related proteins possessing these motifs have been identified. Different members of these families exert their effects on transcription as homodimers and/or in heterodimeric combination with other members of the same family (Lamb and McKnight 1991; Amati and Land 1994). An important question is, How is intrafamily specificity achieved? Baxevanis and Vinson 1993). Here, we describe a genetic approach for analyzing dimerization specificity.

We have designed a genetic selection to facilitate the isolation of mutants that exhibit altered dimerization specificities (ADS mutants). We define an ADS mutant as one that associates more efficiently with itself than with the wild-type parent protein (Fig. 1). Here, we report the isolation of an ADS mutant of the Escherichia coli cAMP receptor protein (CRP).

CRP is a dimeric transcriptional regulator that activates the transcription of many genes in $E$. coli under conditions of glucose depletion (for review, see Kolb et al. 1993). When complexed with cAMP, it binds to one or

${ }^{1}$ Corresponding author. more 22-bp recognition sites located upstream for within) each CRP-responsive promoter. The CRP recognition sites exhibit twofold rotational symmetry, with one CRP monomer contacting each "half-site". Each CRP monomer consists of a large amino domain (residues 1-134) that binds CAMP and mediates dimer formation and a smaller carboxyl domain (residues 139-209) that contains a helix-turn-helix DNA-binding motif (McKay and Steitz 1981; Weber and Steitz 1987) as well as residues implicated in transcriptional activation (Bell et al. 1990; Eschenlauer and Reznikoff 1991; Zhou et al. 1993; Niu et al. 1994). In the three-dimensional crystal structure of the CRP dimer complexed with DNA (Schultz et al. 1991), monomer-monomer contacts are provided primarily by an $\alpha$-helix in the CRP amino domain (termed $\alpha$-helix C); the two $C$ helices wrap around one another in a parallel fashion, forming a short coiled coil (Crick 1953, Cohen and Parry 1990; O'Shea et al. 1991). The cocrystal structure identifies at least 8 residues in each monomer that contact their counterparts along the monomer-monomer interface. Our CRP ADS mutant was found to bear an amino acid substitution involving 1 of these 8 residues.

Results

Construction of a $\lambda c I-C R P$ fusion protein

To examine CRP dimerization, we took advantage of a general approach devised by Hu et al. (1990) and created 


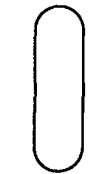

wild type

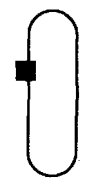

mutant

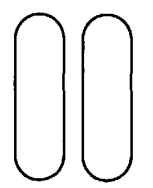

homodimer

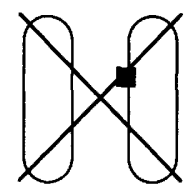

heterodimer

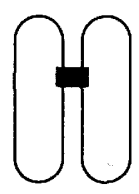

homodimer
Figure 1. Dimerization properties of ADS mutant. The diagram depicts a wild type monomer and an ADS mutant monomer (top), as well as the permitted homodimeric species (bottom). By definition, mutant/mutant homodimer forms preferentially over wild-type/mutant heterodimer.

a fusion protein (called LCF1) consisting of residues 1-132 of $\lambda$ repressor (its DNA-binding domain and linker) connected to residues 2-209 of CRP (Fig. 2A). The CRP moiety confers on LCF1 the ability to dimerize efficiently, thus permitting it to bind to $\lambda$ operators and to render cells immune to $\lambda$ phage (see below). In addition, LCF1 binds to CRP-recognition sites in vivo and in vitro using its ordinary CRP DNA-binding domain /data not shown; also see below|. We also created an LCF1 derivative (called LCF2) bearing a single amino acid substitution (R180G, see Zhang and Ebright 1990) in the CRP DNA-binding domain that prevents the fusion protein from binding to CRP-recognition sites but has no effect on its ability to bind to $\lambda$ operators (data not shown) (Fig. 2A).

\section{Selection strategy}

To isolate CRP ADS mutants we took advantage of the fact that LCF2 can form heterodimers with wild-type CRP. Cells expressing the fusion protein are immune to $\lambda$ phage, whereas cells expressing both the fusion protein and an excess of wild-type CRP are sensitive, presumably because LCF2 is sequestered in CRP/LCF2 heterodimers that can not bind to $\lambda$ operators (Fig. 2B). Under these circumstances, CRP mutants can be selected that are unable to sequester LCF2 in heterodimer form and therefore permit the cells to survive phage challenge. Immune survivors can then be screened for those that contain CRP mutants capable of forming biologically active homodimers able to stimulate transcription from a CRP-dependent promoter.

To carry out this two-step selection/screen procedure, we made use of a single "selection strain" and a pair of compatible vectors directing the expression of LCF2 and CRP. The selection strain (see Materials and methods) bears a deletion removing the chromosomal crp gene and carries a CRP-dependent promoter driving expression of the $l a c Z$ gene. We reasoned that we could identify CRP
ADS mutants by plating doubly transformed selection strain cells doubly transformed with plasmids expressing CRP and LCF2 on medium seeded with $\lambda c \mathrm{I}^{-}$phage and looking for survivors that form blue colonies when streaked on indicator plates containing the chromogenic lactose analog X-gal (see Fig. 2B).

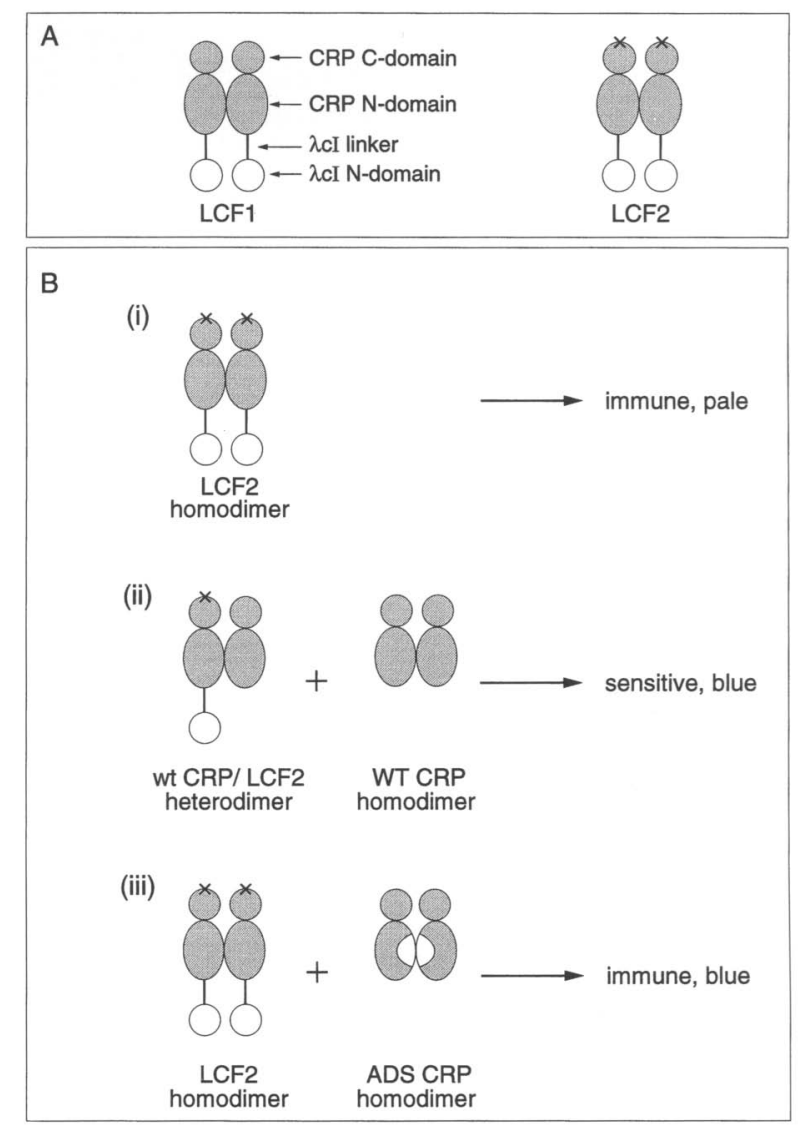

Figure 2. Genetic strategy for isolation of CRP ADS mutants. (A) $\lambda$ cI-CRP fusion (LCF) protein. The fusion protein was created to facilitate the isolation of CRP ADS mutants. LCF1 can bind to both $\lambda$ operators and CRP recognition sites, whereas LCF2 bears a single amino acid substitution in its CRP DNAbinding domain (indicated by the $\times$ signs), and therefore cannot bind to CRP-recognition sites. The abilities of LCF1 and LCF2 to dimerize (and therefore to bind to $\lambda$ operators) do not depend upon cAMP (J. Joung and A. Hochschild, unpubl.). (B) Selection strain cells containing LCF2 only $(i)$ are immune to infecting phage, but form pale colonies on indicator plates because $1 a c Z$ transcription is unstimulated. However, selection strain cells containing LCF2 and an excess of wild-type (wt) CRP (ii) are sensitive to phage because wild-type CRP sequesters LCF2 into LCF2/wt CRP heterodimers. These cells form blue colonies on indicator plates because $1 a c Z$ transcription is stimulated by wild-type CRP homodimer. In contrast, selection strain cells containing LCF2 and an excess of ADS CRP (iii) are immune because ADS CRP does not sequester LCF2 in heterodimer form. These cells also form blue colonies on indicator plates because lacZ transcription is stimulated by ADS CRP homodimer. 


\section{Isolation of $A D S$ mutant candidates}

Mutagenesis was targeted to the region of $c r p$ encoding the amino domain, which contains determinants for dimer formation, using the polymerase chain reaction (PCR). For technical reasons, it was necessary to introduce the mutagenized CRP-encoding plasmid into the selection strain before introducing the plasmid encoding LCF2 (see Materials and methods). Therefore, selection strain cells were transformed with the pools of mutagenized CRP-encoding plasmid, and the resulting transformants were used to make pools of competent cells that were then transformed with the LCF2-expressing plasmid and spread on plates seeded with $\lambda \mathrm{CI}^{-} \mathrm{h} 80$ phage (see Materials and methods). Cells containing $\sim 14,000$ mutant CRP candidates were transformed with the LCF2-encoding plasmid, and five different CRP mutants were identified that permitted the secondary transformants to survive phage challenge (Materials and methods). All five also permitted the cells to form blue colonies on indicator plates containing X-gal.

The plasmids encoding these ADS mutant candidates were purified, and the mutations responsible for the ADS phenotypes were mapped to specific restriction fragments (see Materials and methods). DNA sequencing of these fragments revealed mutations resulting in the following amino acid substitutions: the pair T10A and L113R, S117P, A118P, I112T, and the pair K100R and G141V.

\section{$C R P$ mutants stimulate lacZ transcription in vivo}

To quantify the effects of the five CRP mutants on lacZ transcription from CRP-dependent promoters, we reintroduced the plasmids encoding the CRP mutants into the selection strain (this time without the LCF2-encoding plasmid) and a similar strain bearing a lacZ gene under the control of a promoter with a stronger CRPbinding site. $\beta$-Galactosidase assays were performed, and as shown in Table 1 , all of the mutants stimulated $1 a c Z$ transcription above the basal level measured in the absence of CRP. The two double mutants, CRP[T10A,L113R] and CRP[K100R,G141V], most closely resembled wild-type CRP in their stimulatory abilities. We chose CRP[T10A,L113R], which stimulated lacZ transcription in the selection strain somewhat better than CRP[K100R,G141V], for further analysis.

\section{CRP[T10A, L113R] fails to sequester LCF protein bearing $a$ wild-type dimerization domain but sequesters $L C F$ protein bearing $T 10 A$ and $L 113 R$ substitutions}

Cross-streak immunity tests were performed to reconfirm the inability of CRP[T10A,L113R] to sequester LCF protein containing a wild-type CRP dimerization domain. Cells deleted for the chromosomal $c r p$ gene were transformed with plasmids encoding either wild-type CRP, CRP[T10A,L113R], LCF1 (able to bind to both $\lambda$ operators and CRP-recognition sites), or the relevant
Table 1. Effects of wild-type and mutant CRPs on lacZ transcription from CRP-dependent promoters

\begin{tabular}{lcc}
\hline & \multicolumn{2}{c}{$\beta$-Galactosidase units } \\
\cline { 2 - 3 } Plasmid-encoded protein & $\begin{array}{c}\text { ICB40 } \Delta \text { crp45 } \\
{[\phi \text { mut32] }}\end{array}$ & $\begin{array}{c}\text { JCB40 } \Delta \text { crp45 } \\
{[\phi \text { lac32] }}\end{array}$ \\
\hline No CRP & 122 & 78 \\
wt CRP & 386 & 2780 \\
CRP [T10A, L113R] & 317 & 2400 \\
CRP [K100R, G141V] & 250 & 2340 \\
CRP [I112T] & 215 & 1800 \\
CRP [A118P] & 213 & 1550 \\
CRP [S117P] & 165 & 1230 \\
\hline
\end{tabular}

Cells harboring pHA7E plasmids expressing the various mutant CRPs listed were assayed for $\beta$-galactosidase activity. For the control without CRP, the cells were transformed with plasmid pLR $1 \Delta \mathrm{cI}$.

${ }^{a}$ Transformants of strain JCB40 $\Delta$ crp45[ $\phi$ mut32] (the selection strain) were assayed. Two independent transformants of each type were assayed in duplicate, and the values shown were obtained by averaging the results for the independent transformants. These measurements differed by $<12 \%$ in each case. The same experiment was repeated on a separate occasion and yielded similar results.

${ }^{\mathrm{b}}$ Transformants of strain JCB40Acrp45[\$lac32] were assayed in duplicate, and the values shown are the averages; the duplicate values differed by $<10 \%$, except in one case (CRP [A118P]) where they differed by $18 \%$. The same experiment was repeated several times, and the ratios of the values obtained for CRP [T10A, L113R] and wild-type CRP varied from 0.78 to 1.0 .

pairwise combinations. Cross-streak tests were done using the $\lambda \mathrm{CI}^{-} \mathrm{h} 80$ phage to assess the relative immunities of the various transformants. Table $2 \mathrm{~A}$ shows that cells containing LCF1 and wild-type CRP were sensitive, whereas those containing either LCF1 alone or LCF1 and CRP[T10A,L113R] were immune.

To determine the contribution of each amino acid substitution, we introduced each mutation separately into the wild-type $c r p$ gene and tested for $\lambda$ immunity as before (Table 2A). Mutant CRP[T10A] behaved essentially like wild-type CRP, rendering selection strain cells containing LCF1 sensitive to the phage, whereas mutant CRP[L113R] behaved indistinguishably from the double mutant in this assay. However, because of the physical proximity of T10 and $\mathrm{L} 113$ in the three-dimensional structure of CRP (see Discussion), we chose to retain the double mutant for further analysis.

To further test the idea that CRP[T10A,L113R] is an ADS mutant, we introduced the T10A and L113R substitutions into LCF1, thus generating a new version of the fusion protein called LCF5. If these substitutions alter the dimerization specificity of CRP, then cells containing LCF5 should be immune to infecting $\lambda$ phage in the presence of high levels of wild-type CRP, but not CRP[T10A,L113R], owing to the greater ability of CRP[T10A,L113R] to sequester LCF5 in heterodimer form.

Cells deleted for the chromosomal crp gene were 
Table 2. Results of immunity tests to assess sequestration of LCF1 and LCF5 by wild-type CRP and CRP [T10A, L113R]

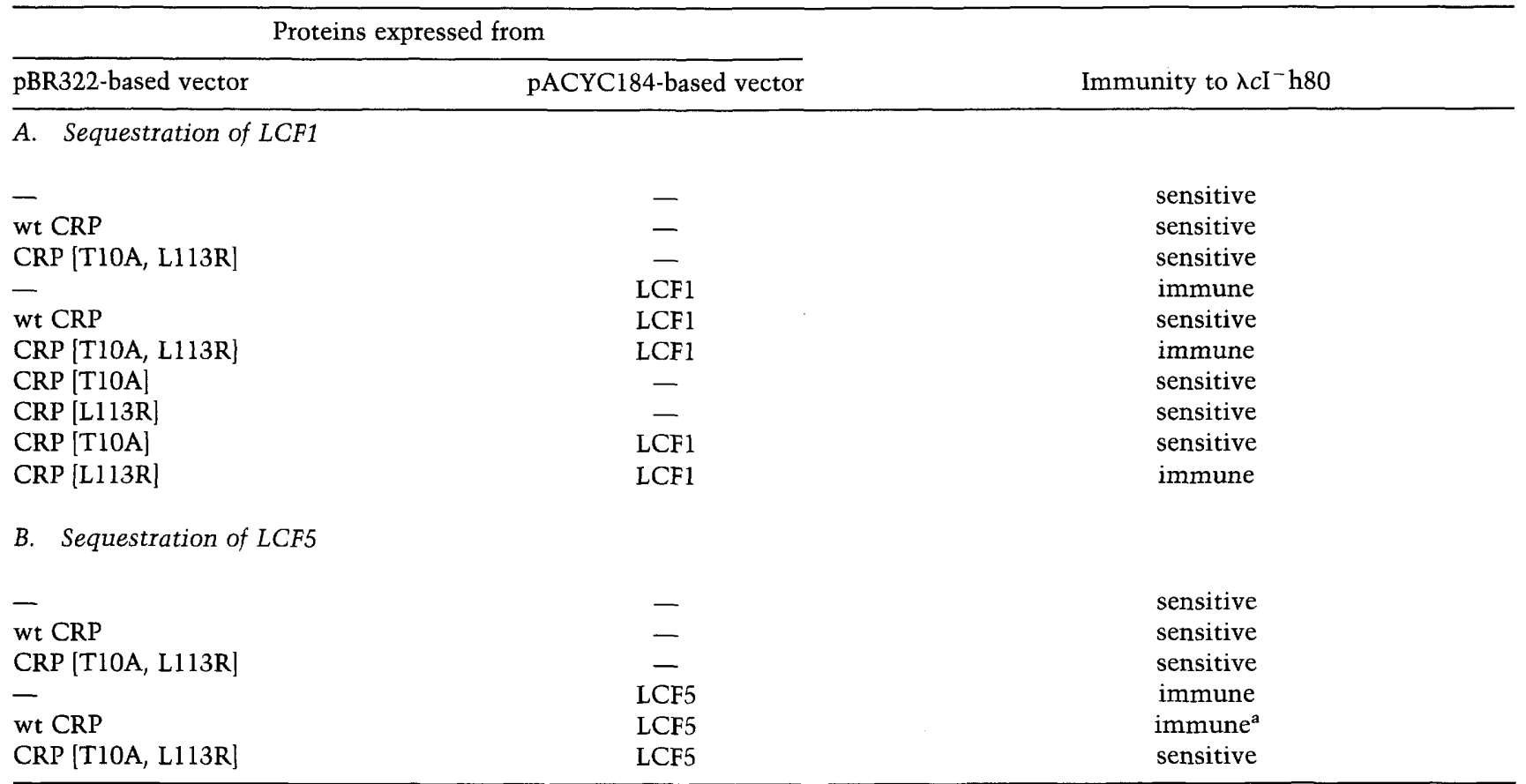

JCB43 $\Delta$ crp $_{39}$ cells containing plasmids expressing the various combinations of proteins shown were tested for their immunity to $\lambda \mathrm{CI}^{-} \mathrm{h} 80$ phage by cross-streak analysis (see Materials and methods). All of the CRP proteins were encoded on derivatives of pHA7E, and the LCF proteins were encoded by pAC-LCF1 or pAC-LCF5. The pBR322-based control vector was pLR1 $\mathrm{cI}$, and the pACYC184based control vector was pAD325. [The cells also contained a low-copy-number plasmid $\left(\mathrm{pP}_{\mathrm{RM}} \Delta-50\right.$; Joung et al. 1994) that is not relevant to this experiment]. Cross-streaks were repeated at least five times with reproducible results. Experiments in $A$ were performed in the absence of IPTG, whereas experiments in $B$ were performed with $3 \mu \mathrm{M}$ IPTG in the plate media. IPTG addition was necessary to express a level of LCF5 sufficient to confer immunity, because the intracellular concentration of LCF5 is lower than that of LCF1 as judged by Western blot analysis (data not shown).

${ }^{a}$ With this transformant, some of the cells in the stripe were lysed by the phage (i.e., the stripe was noticeably thinner after crossing the zone of phage), suggesting that the concentration of LCF5 homodimer in these cells is at the threshold required for immunity.

transformed with either wild-type CRP, CRP[T10A, L113R], LCF5, or the relevant pairwise combinations. Consistent with the prediction, cells containing LCF5 and CRP[T10A,L113R] were sensitive, whereas cells containing LCF5 and wild-type CRP were immune (Table $2 \mathrm{~B}$ ).

Direct visualization of predicted homo- and heterodimeric species by electrophoretic mobility-shift analysis

To obtain direct evidence for the presence of the predicted homo- and heterodimeric protein species in cells containing CRP (either wild-type or T10A,L113R) and fusion protein (either LCF1 or LCF5), we performed electrophoretic mobility-shift analysis using cell extracts (see Materials and methods). We took advantage of the fact that all three species, CRP homodimers, LCF homodimers, and CRP/LCF heterodimers, are expected to bind CRP-recognition sites and to yield complexes that have different mobilities on a native polyacrylamide gel (a general approach first used by Hope and Struhl 1987). We incubated the various extracts with a radiolabeled double-stranded DNA probe bearing a high-affinity CRPbinding site and subjected the samples to gel electropho- resis (Fig. 3A). Extracts prepared from cells expressing only wild-type CRP, only CRP[T10A,L113R], only LCF1, or only LCF5 contained the expected homodimeric DNA-binding species (lanes 2-4,7). Extracts prepared from cells expressing both wild-type CRP and LCF1 contained CRP homodimer and a new species migrating in the region expected for the heterodimer but no detectable LCF1 homodimer (lane 5). In contrast, extracts prepared from cells expressing both CRP[T10A,L113R] and LCF1 contained the two homodimeric species and no detectable heterodimer (lane 6). Extracts prepared from cells expressing both CRP[T10A,L113R] and LCF5 contained CRP homodimer as well as the predicted heterodimeric species (lane 9). This analysis yielded one anomaly: Despite the fact that cells expressing wild-type CRP and LCF5 contained LCF5 homodimer as judged by their immunity to $\lambda$ phage (Table $2 B$ ), we did not detect the LCF5 homodimer (nor any heterodimer) in extracts prepared from these cells (lane 8).

We suspected that the various dimeric species in these extracts were competing for a limited amount of DNA probe under the conditions of the binding assay. To look directly at the relative amounts of CRP and LCF protein in the various extracts, we subjected samples of each extract to denaturing polyacrylamide electrophoresis 


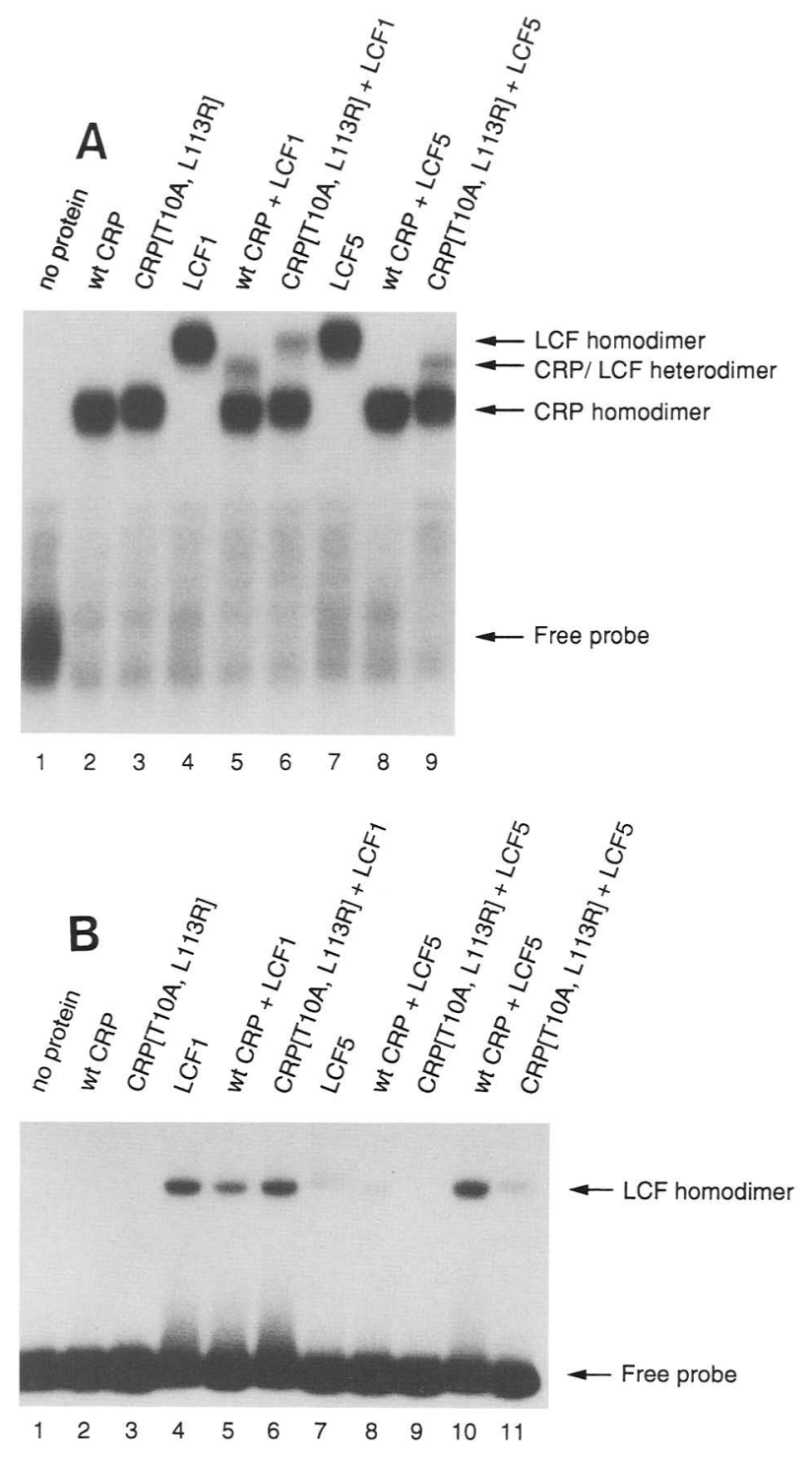

Figure 3. Electrophoretic mobility-shift assays to detect presence of various homo- and heterodimeric species. Electrophoretic mobility-shift assays were performed with cell extracts and an internally labeled DNA fragment bearing a consensus CRP binding site $(A)$, or a single $\lambda$ operator site (see Materials and methods $(B)$. The extracts were prepared from JCB43 $\Delta c r p_{39}$ cells expressing the proteins indicated (see Materials and methods). (A) The amount of total cellular protein in each reaction was $4.8 \mu \mathrm{g}$ except for the reaction loaded in lane 8 , which contained $2.4 \mu \mathrm{g}$; cells expressing wild-type CRP and LCF5 grew more slowly. The experiment was repeated subsequently using $2.4 \mu \mathrm{g}$ of total cellular protein for all reactions and yielded identical results (data not shown). $(B)$ The amount of total cellular protein in each reaction was $270 \mathrm{ng}$ except for the reactions loaded in lanes 10 and 11, which contained $1.35 \mu \mathrm{g}$. In $A$ and $B$, unbound DNA probe and species corresponding to CRP homodimer, CRP/LCF heterodimer, and LCF homodimer bound to DNA are indicated.

and Western blot analysis. This assay revealed (1) that the concentration of each fusion protein (either LCF1 or
LCF5) was the same whether or not the relevant cell extract also contained CRP and (2) that the concentrations of CRP[T10A,L113R] and LCF5 were lower than the concentrations of CRP and LCF1, respectively (data not shown). Thus, cells expressing wild-type CRP together with LCF5 contained less fusion protein relative to CRP than cells expressing any other protein combination, providing a possible explanation for our failure to detect the LCF5 homodimer in extracts prepared from these cells.

To facilitate detection of the LCF5 homodimer, we performed electrophoretic mobility-shift assays using a DNA probe bearing a high-affinity $\lambda$ operator to which only LCF homodimers are expected to bind. Consistent with the results of the immunity tests (Table 2B), this assay revealed that cells expressing wild-type CRP together with LCF5 did contain LCF5 homodimer (Fig. 3B). With this assay we were able to detect $\lambda$ operator binding activity in all extracts that contained fusion protein. As anticipated on the basis of the results of the immunity tests, cells expressing wild-type CRP and LCF5 contained more LCF5 homodimer than cells expressing CRP[T10A,L113R] and LCF5 (cf. lanes 8 and 9 and lanes 10 and 11), whereas cells expressing CRP[T10A,L113R] and LCF1 contained more LCF1 homodimer than cells expressing wild-type CRP and LCF1 (cf. lanes 5 and 6).

\section{Discussion}

We have described the isolation of a CRP mutant [T10A,L113R] altered in its dimerization specificity such that it associates more efficiently with itself than with the dimerization domain of wild-type CRP. This mutant was isolated based on (1) its inability to sequester a $\lambda c I-$ CRP fusion protein in CRP/fusion protein heterodimers and (2) its ability to form homodimers capable of stimulating lacZ transcription from a CRP-dependent promoter.

We further tested our hypothesis that CRP[T10A,L113R] is an ADS mutant by constructing a version of the $\lambda \mathrm{CI}-\mathrm{CRP}$ fusion protein bearing the T10A and L113R substitutions (called LCF5) and comparing the abilities of wild-type CRP and CRP[T10A,L113R] to sequester LCF5 in CRP/LCF5 heterodimers. The construction of LCF5 enabled us to exclude an alternative explanation for the isolation of CRP[T10A,L113R]: namely, that the substitutions T10A and L113R decrease the effective concentration of CRP dimer, either because of an effect on dimerization itself or because of an effect on protein stability. The decrease would have to be significant enough to relieve the sequestration of LCF2 in the selection strain but modest enough to permit mutant homodimers to form. This interpretation of the behavior of the CRP[T10A,L113R] mutant leads to a prediction that is contradicted by our data: namely, that wild-type CRP should be better able than CRP[T10A,L113R] to sequester LCF5. On the contrary, we found that cells expressing wild-type CRP and LCF5 contained more LCF5 homodimer than cells expressing CRP[T10A,L113R] and 
LCF5 as determined by both immunity tests (Table 2B) and electrophoretic mobility-shift analysis (Fig. 3B).

In the case of the four other CRP mutants that we isolated, we have no evidence that they are actually altered in their dimerization specificities. We note that three of the four bear amino acid substitutions within the C $\alpha$-helix. We were surprised initially that among the CRP mutants we isolated, none were completely defective in their abilities to dimerize and hence to stimulate $l a c Z$ transcription in the selection strain cells. However, because $\Delta c r p$ cells grow more slowly than their wildtype counterparts, we speculate that selection strain cells lacking any CRP activity may have failed to form visible colonies under the conditions of our selection.

The crystal structures of CRP reveal that the two C $\alpha$-helices of the monomers interact, wrapping around each other to form a parallel coiled coil (McKay et al. 1982; Schultz et al. 1991). In a coiled-coil structure, each $\alpha$-helix contains 3.5 amino acid residues per turn, and thus, the structure is characterized by a heptad repeat (Crick 1953; for review, see Alber 1992). By convention, the seven different amino acid positions are designated "abcdefg", and the residues that pack most closely against one another along the dimerization interface are assigned to the a and d positions (Fig. 4A). The a and $d$ residues are typically hydrophobic, and each of these residues within one $\alpha$-helix can interact with its counterpart in the other helix (e.g., see O'Shea et al. 1991). In CRP, residue $\mathrm{L} 113$ lies within the $\mathrm{C} \alpha$-helix near its amino-terminal end, and it is the first $d$ position residue in that helix. The crystal structure of the CRP dimer complexed with DNA indicates that the two L113 residues face each other and interact at the monomer-monomer interface (Schultz et al. 1991). Although residue 10 is well separated from residue 113 in the primary sequence of CRP, the crystal structure reveals that T10, which is located at the amino terminus of $\alpha$-helix $A$, closely approaches the amino-terminal end of $\alpha$-helix $C$. In fact, the hydroxyl side chain of T10 forms a hydrogen bond with the carbonyl side chain of D111, the first residue of $\alpha$-helix C. Nevertheless, we found that cells bearing the single mutant CRP $[\mathrm{L} 113 \mathrm{R}]$ and LCF1 were immune to phage infection (Table 2A) and that CRP[L113R] stimulated lacZ transcription from CRP-dependent promoters approximately as efficiently as the double mutant (data not shown). We do not know, therefore, whether the T10A substitution contributes to the ADS phenotype of CRP[T10A,L113R].

CRP is a member of a family of transcriptional regulators defined by amino acid sequence similarities. This family includes the $E$. coli FNR protein as well as related proteins from a variety of other microorganisms (for review, see Spiro and Guest 1990; Kolb et al. 1993). CRP is the only member of this family whose structure has been determined, but sequence alignments suggest that other family members resemble CRP in overall structure (Fig. 4B) (Shaw et al. 1983; Cherfils et al. 1989; Spiro and Guest 1990). In the case of FNR, an iron-binding transcriptional regulator that activates the expression of many genes in response to anaerobiosis, genetic and bio-

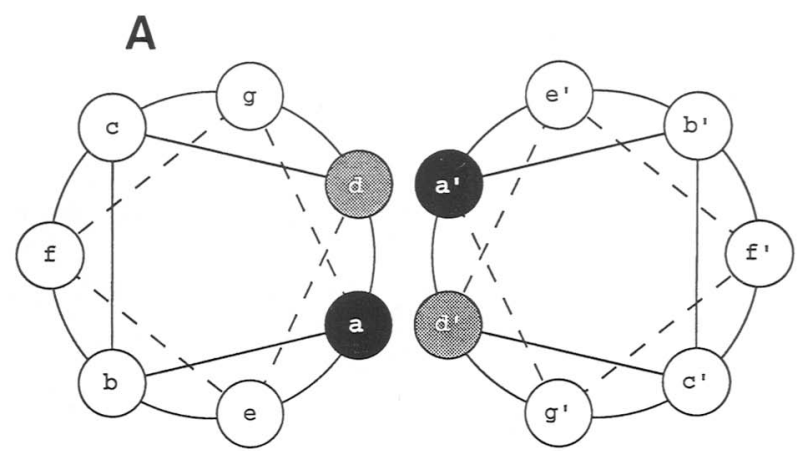

B

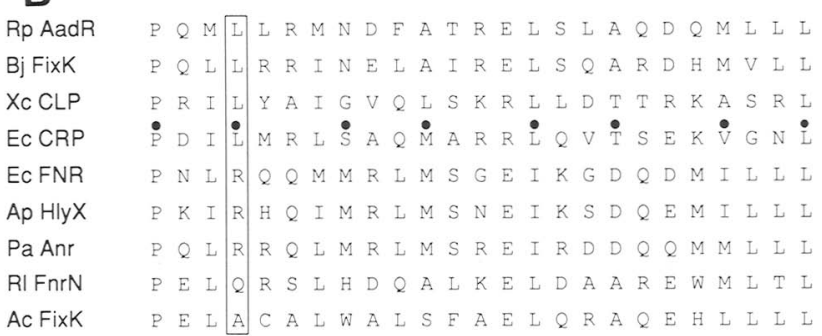

Figure 4. Coiled-coil structure and amino acid sequence alignments. (A) Helical wheel representation of coiled-coil structure. The seven amino acid positions are designated with the letters $a-g$ for one of the $\alpha$-helices, and $a^{\prime}-g^{\prime}$ for the other. Residues at positions $a$ and $a^{\prime}$ pack against each other above the plane of the page, and residues at positions $d$ and $d^{\prime}$ pack against each other below the plane of the page. $(B)$ Amino acid sequence of the $C \alpha$-helix of CRP and aligned sequences from eight related proteins: E. coli (Ec) FNR protein (Shaw et al. 1983), Rhodopseudomonas palustris (Rp) AadR protein (Dispensa et al. 1992), Bradyrhizobium japonicum (Bj) FixK protein (Anthamatten et al. 1992), Xanthomonas campestris (Xc) CLP protein (Dong and Ebright 1992), Actinobacillus pleuropneumoniae (Ap) HlyX protein (MacInnes et al. 1990), Pseudomonas aeruginosa (Pa) Anr protein (Sawers 1991), Rhizobium leguminosarum (Rl) FnrN protein (Colonna-Romano et al. 1990), and Azorhizobium caulinodans (Ac) FixK protein (Kaminski et al. 1991). The sequences are aligned as reported previously (Spiro and Guest 1990; Anthamatten et al. 1992; Dispensa et al. 1992; Dong and Ebright 1992). Shown in line 4 is the amino acid sequence of $\mathrm{CRP}^{\prime}$ 's dimerization region (residues 110-134), the $\mathrm{C} \alpha$-helix extending from residue 111 to 134 . (O) a and d position residues (except P110, which is not part of the $\alpha$-helix) that pack against their counterparts at the monomer-monomer interface. The residues corresponding to $\mathrm{L} 113$ of CRP (the first d position residue in the helix) have been boxed.

chemical evidence implicates a predicted $\mathrm{C} \alpha$-helix counterpart in FNR dimerization (Kiley and Reznikoff 1991; Lazazzera et al. 1993). Alignment of the putative C helix of FNR with that of CRP reveals similar or identical residues corresponding to 5 of $8 \mathrm{CRP}$ residues that participate directly in contacts at the monomer-monomer interface (Fig. 4B).

Strikingly, wild-type FNR bears an arginine residue at the position corresponding to L113 in CRP, raising the possibility that our CRP ADS mutant and FNR may uti- 
lize this arginine in similar fashions. Alignment of the amino acid sequences of seven CRP-related proteins with those of CRP and FNR indicates that at least two other proteins (the HlyX protein of Actinobacillus pleuropneumoniae and the Anr protein of Pseudomonas aeruginosa) also bear an arginine residue at this position (Fig. 4B).

These comparisons suggest that an arginine at the $d$ position closest to the amino-terminal end of the helix is at least compatible with specific coiled-coil formation. Examination of $\alpha$-fibrous proteins that form extended dimeric coiled coils reveals, however, that arginine residues are rarely found at the $\mathrm{d}$ position /Conway and Parry 1990), presumably because their presence destabilizes interhelical interactions. It is possible that an arginine at the amino-terminal $d$ position may function somewhat differently than an arginine at other $d$ positions within such helices. In this regard, we note that $\mathrm{Hu}$ et al. (1990), in their mutational analysis of the GCN4 leucine zipper fused to the $\lambda$ repressor amino domain, found that mutants bearing arginine substitutions at various d positions in the zipper behaved differently: A mutant bearing an arginine at the amino-terminal $\mathrm{d}$ position was capable of homodimerization, whereas mutants bearing an arginine at any of the other d positions were unable to dimerize at all.

Previous studies of leucine zipper dimerization motifs have indicated that residues at the $\mathrm{e}$ and $\mathrm{g}$ positions can dictate dimerization specificity. The e and $g$ residues in leucine zipper sequences are often charged, and interhelical salt bridges involving these residues have been observed (O'Shea et al. 1991; Ellenberger et al. 1992; but see Lumb and Kim 1995). In the case of the Fos and Jun proteins, which preferentially form heterodimers, O'Shea et al. (1992) demonstrated that in the context of a GCN4 leucine zipper peptide, the Fos and Jun e and g residues are sufficient to specify heterodimer formation and that preferential heterodimer formation is driven by destabilizing interhelical electrostatic interactions in the homodimer bearing the Fos e and $\mathrm{g}$ residues (see also O'Shea et al. 1993). Vinson et al. (1993) have also demonstrated the importance of e and $g$ position residues in determining dimerization specificity among bZIP family proteins (see also Krylov et al. 1994). Our isolation of $\mathrm{CRP}[\mathrm{T} 10 \mathrm{~A}, \mathrm{~L} 113 \mathrm{R}]$ as an ADS mutant indicates that the identity of at least one d position residue can also dictate dimerization specificity.

The genetic method we have described should facilitate the isolation of ADS mutants of other dimeric transcriptional regulators from both prokaryotes and eukaryotes. ADS mutants may provide useful biological reagents in a variety of situations. For example, experiments that involve the introduction of a particular mutant protein into cells are often complicated by the association of the mutant protein with one or more endogenous proteins, resulting in the formation of unwanted heterodimers. The use of ADS mutants could simplify the analysis under such circumstances. ADS mutants may also provide helpful tools for determining whether a given protein exerts a specific effect as a homodimer or, alternatively, in heterodimeric combination with another partner in the cell.

The isolation of ADS mutants of other proteins, whether they function as homodimers or heterodimers, should provide useful insight into the amino acid interactions that dictate the relevant dimerization specificities. As the approach is genetic, our strategy should permit the analysis of proteins for which no structural information is available. A variation of the approach should also permit the isolation of ADS mutants that have lost the ability to associate with a particular partner while retaining the ability to form biologically active homodimers.

\section{Materials and methods}

General techniques

DNA sequencing and $\beta$-galactosidase assays were performed as described previously (Joung et al. 1993). Site-directed mutagenesis was performed using the method of Kunkel et al. (1987).

\section{Bacterial strains}

The selection strain was constructed by lysogenizing strain JCB40 [F ${ }^{-} \Delta($ gpt-proAB-arg-lac)XIII zaj::Tn10] (Joung et al. 1993 ) with a $\lambda$ phage derivative designated $\phi$ Mut32 (see below), identifying lysogens bearing a single prophage, and then deleting the chromosomal crp gene by transducing the strain to streptomycin resistance with $\mathrm{Pl}^{\text {vir }}$ phage grown on strain

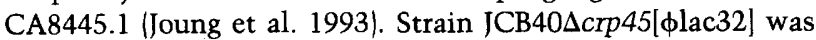
constructed in an analogous manner. Strain JCB43 $\Delta c r p_{39}\left(\mathrm{~F}^{-}\right.$, $\lambda^{-}, \operatorname{lacZ}^{-}, \Delta c r p_{39}$ ) has been described previously (Joung et al. 1994).

\section{Phages}

$\phi M u t 32$ This phage $\lambda$ derivative contains a P22 immunity region and also bears a lac $Z$ gene whose expression is driven by a modified version of the lacPl promoter designated the "weak site template" that has been described previously (Joung et al. 1993). This lac promoter contains a point mutation within its CRP-binding site that reduces the ability of CRP to stimulate transcription $\sim 10$-fold.

$\phi$ lac32 This phage $\lambda$ derivative is identical to $\phi$ Mut32 except that it bears an essentially wild-type lacPl promoter (Joung et al. 1993).

$\lambda \mathrm{cI} I^{-}$and $\lambda \mathrm{cI} I^{-} h 80$ These phage $\lambda$ clear mutants differ in their host ranges such that the h80 phage is able to infect cells that do not express the lamB receptor.

\section{Plasmids}

pHA7E Plasmid pHA7E, a derivative of plasmid pHA7 /Aiba et al. 1982), was used to express high levels of wild-type CRP and was constructed as follows: First, the unique EagI site within the vector sequences of plasmid pHA7 was removed by digesting it with EagI, blunting the ends with the Klenow fragment of DNA polymerase I (Klenow), and then inserting a single $\mathrm{XbaI}$ linker (New England Biolabs, linker 1083) between the blunted ends. Second, a point mutation was introduced into the crp gene at amino acid position 140 (ACG to ACC) that creates an EagI 
restriction site without changing the amino acid specified by that codon (see below).

pLCF1 To generate a plasmid encoding the desired LCF1 protein, it was first necessary to introduce two changes into the $c r p$ gene: (1) a point mutation at CRP amino acid 140 (ACG to ACC) that creates an EagI restriction site without changing the amino acid specified by the codon at that position and $\langle 2|$ four point mutations within and just upstream of the crp initiation codon that create a SalI restriction site and that eliminate the $c r p$ initiation codon. To permit site-directed mutagenesis of the crp gene, an $\sim 1-\mathrm{kb}$ HindIII-EcoRI fragment encoding wild-type CRP from plasmid pHA7 (Aiba et al. 1982) was inserted between the unique HindIII and EcoRI sites in phagemid EMBL9+ (Dente et al. 1983) to create plasmid EM9HA7. EM9HA7 was then digested with EcoRI, the ends were blunted with Klenow, and a $B a m H I$ linker was inserted at this position to generate plasmid EM9HA7-Bam. The insertion of this linker introduces a unique $B a m H I$ restriction site but also regenerates two flanking EcoRI sites. Two oligonucleotides, 5'-GCGGCCGGTCACGTCG-3' and 5'-CCAAGCACCGTCGACGGTTATCC$3^{\prime}$, were then used to introduce the desired mutations (boldface) into the crp gene on EM9HA7-Bam. To fuse CRP residues 2-209 to $\lambda$ cI residues $1-132$, the resulting plasmid was digested with SalI and BamHI and the fragment containing CRP coding sequences ligated to the large SalI-BamHI fragment of plasmid pJH370 (Hu et al. 1990) to generate plasmid pLCF1. pLCF1 contains a pBR322 origin of replication, provides ampicillin resistance, and directs expression of the LCFl fusion protein under the control of the lacUV5 promoter.

pLCF2 The pLCF2 plasmid is identical to plasmid pLCF1 except for the presence of a mutation that changes amino acid 180 of CRP from an Arg to a Gly (Zhang and Ebright 1990), which was introduced using the oligonucleotide 5'-GGTTTCACCAGAACA-3'.

pAC-LCF1 and pAC-LCF2 Plasmids pAC-LCF1 and pACLCF2 were used to express LCF1 and LCF2, respectively. pACLCF1 and pAC-LCF2 were constructed by digesting plasmids pLCF1 and pLCF2, respectively, with EcoRI, blunting the ends with Klenow, and isolating the $\sim 1.4-\mathrm{kb}$ fragments containing the lacUV5 promoter and coding sequences for the fusion proteins. These blunt-ended fragments were each ligated to plasmid pAD325 (Derman et al. 1993; see below) that had been linearized with BamHI and treated with Klenow to blunt the ends. Recombinants that contained a single insertion in the same orientation as the disrupted tetracycline gene were identified and designated pAC-LCF1 and pAC-LCF2. Note that these plasmids provide chloramphenicol resistance and also encode a $l a c I^{\mathrm{q}}$ gene product that can repress the lacUV5 promoter that controls LCF protein expression.

pAC-LCF5 Plasmid pAC-LCF5 was used to express LCF5 and is identical to pAC-LCF1 except that it contains the T10A (GCC to ACC) and L113R (CTG to CGG) mutations within the crp portion of the fusion protein gene.

Other plasmids Plasmid pAD325 is a derivative of plasmid pACYC184 that contains a lacl ${ }^{q}$ gene (Derman et al. 1993). The control plasmid pLRl $\Delta \mathrm{cI}$ encodes neither functional $\lambda c \mathrm{I}$ nor CRP (Whipple et al. 1994). Plasmid pMH-Cons/RM93 contains a consensus CRP-binding site, 5'-AAATGTGATCTAGATCACATTT-3', derived from plasmid $\mathrm{pP}_{\mathrm{RM}} \Delta-50 / \mathrm{CRP}$ (Joung et al. 1994). Plasmid pBS-O ${ }_{L} 1$ (Hayashibara and Verdine 1992) con- tains a single high-affinity $\lambda$ operator $\left(5^{\prime}\right.$-TATCACCGCCAGTGGTA-3').

\section{Genetic selection}

Mutagenesis of plasmid pHA7E To randomly mutagenize the crp gene encoded by plasmid pHA7E, we used the PCR mutagenesis technique of Ebright and colleagues (Zhou et al. 1991). We targeted the mutagenesis to the portion of the gene encoding the amino-terminal domain of CRP by using primers that flank the unique HindIII and EagI restriction sites found in pHA7E. The resulting PCR product was digested with HindIII and EagI restriction enzymes and purified by polyacrylamide gel electrophoresis, yielding mutagenized fragments of DNA encoding amino acids 1-140 of CRP. Eight independently generated pools of such fragments were ligated to vector sequences from plasmid pHA $7 \mathrm{E}$ that had been digested with HindIII and EagI. Each ligation reaction thus contains a pool of regenerated plasmids with random mutations distributed between the HindIII and EagI sites.

Selection procedure for isolating CRP ADS mutant candidates Strains deleted for crp are not as healthy as $\mathrm{crp}^{+}$strains. We therefore anticipated that cells containing LCF2 only, which retains some residual CRP DNA-binding activity, would be under a selective pressure to increase the level of LCF2 expression. To avoid enriching for such mutants prior to the introduction of pHA7E, we first transformed selection strain cells with pools of mutated pHA7E plasmid. The resulting transformants were harvested and made competent, and these cells were then transformed with pAC-LCF2 and plated on lawns of $\mathrm{ACI}^{-}$phage. This selection procedure was performed with eight independently derived pools of mutated pHA7E plasmid.

In the case of the first pool of mutated pHA7E plasmid, we obtained $\sim 300$ transformants using selection strain cells made competent by a divalent cation procedure. These transformants were pooled and transformed with plasmid pAC-LCF2 and plated on TYE agar containing antibiotics $(100 \mu \mathrm{g} / \mathrm{ml}$ carbenicillin and $30 \mu \mathrm{g} / \mathrm{ml}$ of chloramphenicol) and seeded with $\mathrm{\lambda cI}^{-}$ and $\lambda \mathrm{CI}^{-} \mathrm{h} 80$ phage ( $10^{9} \mathrm{pfu}$ each). Four isolates of mutant CRP[T10A,L113R] were recovered. Upon retransforming the plasmid encoding this mutant into selection strain cells containing LCF2, we found that the doubly transformed cells were immune to $\lambda c \mathrm{I}^{-} \mathrm{h} 80$ but not to $\lambda \mathrm{CI}^{-}$phage, suggesting that insufficient LCF2 is expressed under these experimental conditions to render the selection strain cells immune to $\lambda \mathrm{cI}^{-}$phage. (Note that although we were able to determine in advance that selection strain cells containing pAC-LCF2 only were immune to $\mathrm{ACI}^{-} \mathrm{h} 80$ infection, we could not assess whether those cells were also immune to $\lambda \mathrm{CI}^{-}$infection because this phage enters $E$. coli using the lamB receptor and the selection strain fails to express this receptor efficiently owing to the deletion of its chromosomal crp gene.) We hypothesize that under the conditions of the initial plating we simultaneously selected for a CRP mutant to relieve LCF2 sequestration and also a bacterial cell mutant to provide resistance to $\lambda \mathrm{CI}^{-}$(host range $\lambda$ ). Thus, in our subsequent selection experiments, we chose to use only $\lambda c \mathrm{I}^{-} \mathrm{h} 80$ phage $\left(2 \times 10^{10} \mathrm{pfu}\right)$.

In the case of each of the seven other pools of mutated pHA7E plasmid, we obtained $\sim 2000$ primary transformants by electroporating the selection strain cells. Two of the pools yielded one isolate of CRP[K100R,G14IV] and CRP[I112T], respectively, and one pool yielded four isolates of CRP[S117P] and one isolate of CRP[A118P]. We hypothesize that multiple isolations of the same mutation within a given pool could be attributable either 
to amplification of a mutation during PCR mutagenesis or to the replication of mutants that occurs when the primary transformants (containing mutagenized pHA7E) are grown into colonies prior to their preparation as competent cells.

Identification of CRP ADS mutant candidates Colonies that survived the selection process were purified and retested for $\lambda$ immunity by cross-streak analysis (see below). To determine which of the candidate colonies that passed the cross-streak test were immune owing to mutations in the CRP-encoding plasmid, pHA7E DNA was purified from each candidate and then cotransformed with pAC-LCF2 into selection strain cells, and the resulting transformants were then retested for immunity by cross-streak. Transformants that remained immune at this step were then streaked on media containing X-gal to assess colony color. All candidates remaining at this step yielded blue colonies of varying intensity. In the next step, we determined that both phenotypes (immunity and blue color) were linked to the HindIII-EagI fragment within each of the mutant pHA7E plasmids except for one. In the case of the one exception /the plasmid encoding CRP[K100R,G141V]l, the phenotypes could not be mapped to the HindIII-EagI fragment because one of the mutations eliminates the EagI site; we therefore mapped the phenotypes to the HindIII-EcoRI fragment that contains the entire $c r p$ gene. After the mutant phenotypes were mapped, we sequenced the appropriate fragments to identify the mutations.

\section{Cross-streak immunity tests}

Cross-streak tests were performed to assess immunity to $\lambda$ phage. Phage $\left(\sim 10^{9} \mathrm{pfu}\right)$ were striped down the center of an agar plate, and cells from single colonies were streaked perpendicularly across the phage stripe with a toothpick.

\section{Electrophoretic mobility-shift analysis}

Labeling of DNA fragments We used PCR to generate two internally ${ }^{32} \mathrm{P}$-labeled DNA fragments containing either a consensus CRP-binding site or the $\lambda$ operator $\mathrm{O}_{\mathrm{L}} 1$. Plasmid $\mathrm{pMH}$ Cons/RM93 and two primers, 5'-TAGAGCACCGGGGATC-3' and 5'-CCTATAAAAATAGGCGTATCACGAGGCCCT $\left.3^{\prime}\right)$, were used to amplify a 104-bp DNA fragment containing the CRP-binding site. To label this DNA fragment, $150 \mu \mathrm{Ci}$ of $\left[\alpha^{-32}\right.$ P]dTTP (3000 Ci/mmole) was used with $200 \mu \mathrm{M}$ dGTP, dATP, and dCTP. Plasmid pBS- $\mathrm{O}_{\mathrm{L}} 1$ (Hayashibara and Verdine 1992) and two primers, (5'-AGCGGATAACAATTTCACACAGGA-3' and 5'-CGCCAGGGTTTTCCCAGTCACGAC-3', were used to amplify a 233-bp DNA fragment containing the $\lambda$ operator. To label this DNA fragment, $20 \mu \mathrm{Ci}$ of $\left[\alpha^{-{ }^{32}} \mathrm{P}\right] \mathrm{dATP}$ (3000 Ci/mmole) was used with $200 \mu \mathrm{M} \mathrm{dGTP,} \mathrm{dCTP,} \mathrm{and} \mathrm{dTTP}$ and $20 \mu \mathrm{M}$ dATP. The desired fragments were purified after separation by polyacrylamide gel electrophoresis.

Preparation of extracts Whole-cell extracts were prepared using a modified version of a protocol provided by Dr. Simon Lynch (Harvard University, Cambridge, MA). For these experiments, we utilized the same set of JCB $43 \Delta \operatorname{crp}_{39}$ transformants as were subjected to cross-streak analysis in Table 2A (lines 1-6) and Table 2B (lines 4-6). Transformants were grown to late log phase $\left(\mathrm{OD}_{600}\right.$ of $\left.0.5-1.0\right)$ in LB supplemented with $25 \mu \mathrm{M} \mathrm{IPTG}$, $35 \mu \mathrm{g} / \mathrm{ml}$ of tetracycline, $50 \mu \mathrm{g} / \mathrm{ml}$ of carbenicillin, and $30 \mu \mathrm{g} /$ $\mathrm{ml}$ of chloramphenicol. Cells from $10 \mathrm{ml}$ of culture were harvested and resuspended immediately in $100 \mu \mathrm{l}$ of ice-cold solution A $(25 \%$ sucrose, $40 \mathrm{~mm}$ Tris at $\mathrm{pH} 8.0,1 \mathrm{~mm}$ EDTA, 1 $\mathrm{mg} / \mathrm{ml}$ of lysozyme, $2 \mathrm{mM} \mathrm{cAMP}$ ) and left on ice for 10 minutes. One hundred microliters of ice-cold solution B (10 mM Tris at pH 8.0, $60 \mathrm{mM} \mathrm{MgCl}_{2}, 0.9 \%$ Brij-58, $2 \mathrm{mM}$ cAMP) was then added, and the entire sample was mixed gently and returned to ice for $5 \mathrm{~min}$. The cell debris was removed by centrifugation, and $140 \mu \mathrm{l}$ of supernatant was removed, frozen rapidly, and stored at $-80^{\circ} \mathrm{C}$. The concentration of total protein in each extract was determined by Bradford assay, and the extracts were diluted, if necessary, using a 1:1 mixture of solutions A and B.

Electrophoretic mobility-shift assays The assays with the consensus CRP-binding site and the $\lambda$ operator were performed in essentially the same manner, except that the CRP binding reactions contained cAMP and the $\lambda$ repressor binding reactions contained $\mathrm{CaCl}_{2}$. Cell extract $(3 \mu \mathrm{l})$ was preincubated on ice with $2 \mu \mathrm{l}$ of nucleic acid cocktail $10.5 \mathrm{mg} / \mathrm{ml}$ of poly[d(I-C)], 0.5 $\mathrm{mg} / \mathrm{ml}$ of poly[d(A-T)], $340 \mu \mathrm{g} / \mathrm{ml}$ of chick blood DNA) for 20 min. Five microliters of labeled DNA cocktail $(20 \mathrm{~mm}$ Tris at pH $8.0,6 \mathrm{~mm}$ EDTA, $2 \mathrm{mg} / \mathrm{ml}$ of tRNA, $20 \%$ glycerol, $2 \mathrm{mg} / \mathrm{ml}$ of bovine serum albumin, fraction $\mathrm{V}, 91 \mu \mathrm{g} / \mathrm{ml}$ of chick blood DNA, 4 mM dithiothreitol, labeled DNA fragment, and either $400 \mu \mathrm{M}$ cAMP or $50 \mathrm{mM} \mathrm{CaCl}_{2}$ ) was then added; the entire reaction was gently mixed and returned to ice for $1 \mathrm{hr}$. The reaction was loaded onto a running $10 \%$ native polyacrylamide gel (acrylamide/bisacrylamide ratio 75:1) containing $1 \times \mathrm{TBE}$ and either $200 \mu \mathrm{M}$ cAMP or $2 \mathrm{mM} \mathrm{CaCl}_{2}$ in both the gel and the running buffer. The gel was loaded and run at a constant voltage of $420 \mathrm{~V}$ and kept between $20^{\circ} \mathrm{C}$ and $25^{\circ} \mathrm{C}$ using a refrigerated, circulating water bath apparatus. The gel was exposed to Kodak $\mathrm{X}$-Omat AR film for autoradiography.

\section{Acknowledgments}

We thank Jim Hu for plasmid pJH370, Ashley Wivel for constructing plasmid pLCF2, Simon Lynch for providing the wholecell extract procedure, Todd Arnold for providing the DNA labeling protocol, and Vladimir Podolny and Ana Pis-Lopez for technical assistance. We also thank Mark Ptashne for helpful comments on the manuscript and Tom Ellenberger, Mark Rould, Carl Pabo, and Pehr Harbury for their invaluable assistance in visualizing and interpreting the CRP/DNA cocrystal structure. This work was supported by the National Science Foundation Presidential Young Investigator Award to A.H.

The publication costs of this article were defrayed in part by payment of page charges. This article must therefore be hereby marked "advertisement" in accordance with 18 USC section 1734 solely to indicate this fact.

\section{Note added in proof}

Two recent papers [Bunker and Kingston, NARA 23: 269 (1995) and Marchetti et al., I. Mol. Biol. 248: 541 (1995)] have also reported the sequestration of fusion proteins containing the amino domain of $\lambda c \mathrm{I}$ and a heterologous dimerization domain in heterodimers that cannot bind to $\lambda$ operators.

\section{References}

Aiba, H., S. Fujimoto, and N. Ozaki. 1982. Molecular cloning and nucleotide sequencing of the gene for E. coli cAMP receptor protein. Nucleic Acids Res. 10: 1345-1361.

Alber, T. 1992. Structure of the leucine zipper. Curr. Opin. Genet. Dev. 2: 205-210.

Amati, B. and H. Land. 1994. Myc-Max-Mad: A transcription factor network controlling cell cycle progression, differenti- 
ation and death. Curr. Opin. Genet. Dev. 4: 102-108.

Anthamatten, D., B. Scherb, and H. Hennecke. 1992. Characterization of a fixLJ-regulated Bradyrhizobium japonicum gene sharing similarity with the Escherichia coli fnr and Rhizobium meliloti fixK genes. I. Bacteriol. 174: 2111-2120.

Baxevanis, A.D. and C.R. Vinson. 1993. Interactions of coiled coils in transcription factors: Where is the specificity? Curr. Opin. Genet. Dev. 3: 278-285.

Bell, A., K. Gaston, R. Williams, K. Chapman, A. Kolb, H. Buc, S. Minchin, J. Williams, and S. Busby. 1990. Mutations that alter the ability of the Escherichia coli cyclic AMP receptor protein to activate transcription. Nucleic Acids. Res. 18: $7243-7250$.

Cherfils, J., J.-F. Gibrat, J. Levin, J. Batut, and D. Kahn. 1989. Model-building of Fnr and FixK DNA-binding domains suggests a basis for specific DNA recognition. I. Mol. Recog. 2: 114-121.

Cohen, C. and D.A.D. Parry. 1990. $\alpha$-Helical coiled-coils and bundles: How to design an $\alpha$-helical protein. Proteins $7: 1-$ 15.

Colonna-Romano, S., W. Arnold, A. Scluter, P. Boistard, A. Puhler, and U. Priefer. 1990. An Fnr-like protein encoded in Rhizobium leguminosarum biovar viciae shows structural and functional homology to Rhizobium meliloti FixK. Mol. \& Gen. Genet. 223: 138-147.

Conway, J.F. and D.A.D. Parry. 1990. Structural features in the heptad substructure and longer repeats of two-stranded $\alpha$-fibrous proteins. Int. J. Biol. Macromol. 12: 328-334.

Crick, F.H.C. 1953. The packing of $\alpha$-helices: Simple coiled coils. Acta Crystalogr. 6: 689-697.

Dente, L., G. Cesareni, and R. Cortese. 1983. pEMBL: A new family of single stranded plasmids. Nucleic Acids Res. 11: 1645-1655.

Derman, A.I., J.W. Puziss, P.J. Bassford Jr., and J. Beckwith. 1993. A signal sequence is not required for protein export in prlA mutants of Escherichia coli. EMBO I. 12: 879-888.

Dispensa, M., C.T. Thomas, M.-K. Kim, J.A. Perrotta, J. Gibson, and C.S. Harwood. 1992. AadR regulates anaerobic aromatic acid degradation. I. Bacteriol. 174: 5803-5813.

Dong, Q.P. and R.H. Ebright. 1992. DNA binding specificity and sequence of Xanthomonas campestris catabolite gene activator protein-like protein. I. Bacteriol. 174: 5457-5461.

Ellenberger, T.E., C.J. Brandl, K. Struhl, and S.C. Harrison. 1992. The GCN4 basic region leucine zipper binds DNA as a dimer of uninterrupted $\alpha$ helices: Crystal structure of the proteinDNA complex. Cell 71: 1223-1237.

Ellenberger, T., D. Fass, M. Arnaud, and S.C. Harrison. 1994. Crystal structure of transcription factor E47: E-box recognition by a basic region helix-loop-helix dimer. Genes \& Dev. 8: $970-980$.

Eschenlauer, A.C. and W.S. Reznikoff. 1991. Escherichia coli catabolite gene activator protein mutants defective in positive control of lac operon transcription. I. Bacteriol. 173: 5024-5029.

Ferré-D'Amaré, A.R., G.C. Prendergast, E.B. Ziff, and S.K. Burley. 1993. Recognition by Max of its cognate DNA through a dimeric b/HLH/Z domain. Nature 363: 38-45.

Hayashibara, K.C. and G.L. Verdine. 1992. Template-directed interference footprinting of cytosine contacts in a proteinDNA complex: Potent interference by 5 -Aza- $2^{\prime}$-deoxycytidine. Biochemisty 31: 11265-11273.

Hope, I.A. and K. Struhl. 1987. GCN4, a eukaryotic transcriptional activator protein, binds as a dimer to target DNA. $E M B O$ I. 6: 2781-2784.

Hu, J.C., E.K. O'Shea, P.S. Kim, and R.T. Sauer. 1990. Sequence requirements for coiled-coils: Analysis with $\lambda$ repressor-
GCN4 leucine zipper fusions. Science 250: 1400-1403.

Joung, J.K., L.U. Le, and A. Hochschild. 1993. Synergistic activation of transcription by the Escherichia coli cAMP receptor protein. Proc. Natl. Acad. Sci. 90: 3083-3087.

Joung, J.K., D.M. Koepp, and A. Hochschild. 1994. Synergistic activation of transcription by bacteriophage $\lambda \mathrm{cI}$ protein and E. coli cAMP receptor protein. Science 265: 1863-1866.

Kaminski, P.A., K. Mandon, F. Arigoni, N. Desnoues, and C. Elmerich. 1991. Regulation of nitrogen fixation in Azorhizobium caulinodans: Identification of a fixK-like gene, a positive regulator of nifA. Mol. Microbiol. 5: 1983-1991.

Kiley, P.J. and W.S. Reznikoff. 1991. Fnr mutants that activate gene expression in the presence of oxygen. I. Bacteriol. 173: $16-22$.

Kolb, A., S. Busby, H. Buc, S. Garges, and S. Adhya. 1993. Transcriptional regulation by cAMP and its receptor protein. Annu. Rev. Biochem. 62: 749-795.

Krylov, D., I. Mikhailenko, and C. Vinson. 1994. A thermodynamic scale for leucine zipper stability and dimerization specificity: e and $\mathrm{g}$ interhelical interactions. EMBO $\mathrm{I}$. 13: 2849-2861.

Kunkel, T.A. 1987. Rapid and efficient site-specific mutagenesis without phenotypic selection. Proc. Natl. Acad. Sci. 82: $488-492$

Lamb, P. and S.L. McKnight. 1991. Diversity and specificity in transcriptional regulation: The benefits of heterotypic dimerization. Trends Biochem. Sci. 16: 417-422.

Landschultz, W.H., P.F. Johnson, and S.L. McKnight. 1988. The leucine zipper: A hypothetical structure common to a new class of DNA binding protein. Science 240: 1759-1764.

Lazazzera, B.A., D.M. Bates, and P.J. Kiley. 1993. The activity of the Escherichia coli transcription factor FNR is regulated by a change in oligomeric state. Genes \& Dev. 7: 1993-2005.

Lumb, K.J. and P.S. Kim. 1995. Measurements of interhelical electrostatic interactions in the GCN4 leucine zipper. Science 268: 436-439.

MacInnes, J., J. Kim, C.-J. Lian, and G. Soltes. 1990. Actinobacillus pleuropneumoniae hlyX gene homology with the fnr gene of Escherichia coli. I. Bacteriol. 172: 4587-4592.

McKay, D.B. and T.A. Steitz. 1981. Structure of catabolite gene activator protein at $2.9 \AA$ resolution suggests binding to lefthanded B-DNA. Nature 290: 744-749.

McKay, D.B., I.T. Weber, and T.A. Steitz. 1982. Structure of catabolite activator protein at $2.9-\AA$ resolution. I. Biol. Chem. 257: 9518-9524.

Niu, W., Y.H. Zhou, Q.P. Dong, Y.W. Ebright, and R.H. Ebright. 1994. Characterization of the activating region of Escherichia coli catabolite gene activator protein (CAP) I: Saturation and alanine-scanning mutagenesis. J. Mol. Biol. 243: 595602.

O'Shea, E.K., J.D. Klemm, P.S. Kim, and T. Alber. 1991. X-ray structure of the GCN4 leucine zipper, a two-stranded, parallel, coiled-coil. Science 254: 539-544.

O'Shea, E.K., R. Rutkowski, and P.S. Kim. 1992. Mechanism of specificity in the Fos-Jun oncoprotein heterodimer. Cell 68: 699-708.

O'Shea, E.K., K.J. Lumb, and P.S. Kim. 1993. Peptide "Velcro": Design of a heterodimeric coiled coil. Current Biol. 3: 658667.

Sawers, R.G. 1991. Identification and molecular characterization of a transcriptional regulator from Pseudomonas aeruginosa PAOl exhibiting structural and functional similarity to the FNR protein of Escherichia coli. Mol. Microbiol. 5: 1469-1481.

Schultz, S.C., G.C. Shields, and T.A. Steitz. 1991. Crystal structure of a CAP-DNA complex: The DNA is bent by $90^{\circ}$. Sci- 
ence 253: 1001-1007.

Shaw, D.J., D.W. Rice, and J.R. Guest. 1983. Homology between $\mathrm{CAP}$ and Fnr, a regulator of anaerobic respiration in Escherichia coli. J. Mol. Biol. 166: 241-247.

Spiro, S. and J.R. Guest. 1990. FNR and its role in oxygen-regulated gene expression in Escherichia coli. FEMS Microbiol. Rev. 75: 399-428.

Vinson, C.R., T. Hai, and S.M. Boyd. 1993. Dimerization specificity of the leucine zipper-containing bZIP motif on DNA binding: Prediction and rational binding. Genes \& Dev. 7: 1047-1058.

Weber, I.T. and T.A. Steitz. 1987. Structure of a complex of catabolite gene activator protein and cyclic AMP refined at 2.5 Å resolution. J. Mol. Biol. 198: 31 l-326.

Whipple, F.W., N.H. Kuldell, L.A. Cheatham, and A. Hochschild. 1994. Specificity determinants for the interaction of $\lambda$ repressor and P22 repressor dimers. Genes \& Dev. 8: 12121223.

Zhang, X. and R.H. Ebright. 1990. Identification of a contact between arginine- 180 of the catabolite gene activator protein (CAP) and base pair 5 of the DNA site in the CAP-DNA complex. Proc. Natl. Acad. Sci. 87: 4717-4721.

Zhou, Y., X. Zhang, and R.H. Ebright. 1991. Random mutagenesis of gene-sized DNA molecules by use of PCR with Taq DNA polymerase. Nucleic Acids Res. 19: 6052.

. Identification of the activating region of catabolite gene activator protein (CAP)-isolation and characterization of mutants of CAP specifically defective in transcription activation. Proc. Natl. Acad. Sci 90: 6081-6085. 


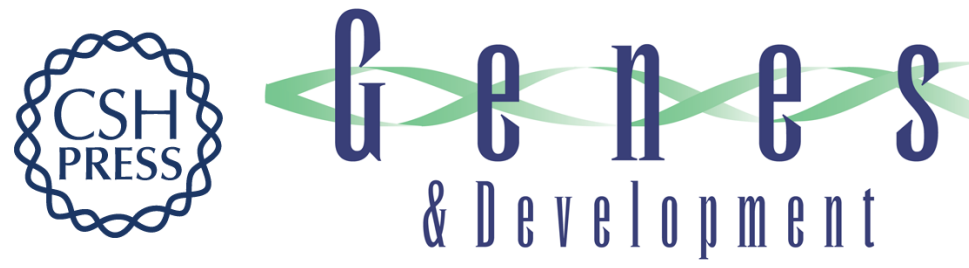

\section{Genetic strategy for analyzing specificity of dimer formation: Escherichia coli cyclic AMP receptor protein mutant altered in its dimerization specificity.}

J K Joung, E H Chung, G King, et al.

Genes Dev. 1995, 9:

Access the most recent version at doi:10.1101/gad.9.23.2986

References This article cites 48 articles, 19 of which can be accessed free at:

http://genesdev.cshlp.org/content/9/23/2986.full.html\#ref-list-1

License

Email Alerting

Service

Receive free email alerts when new articles cite this article - sign up in the box at the top right corner of the article or click here.

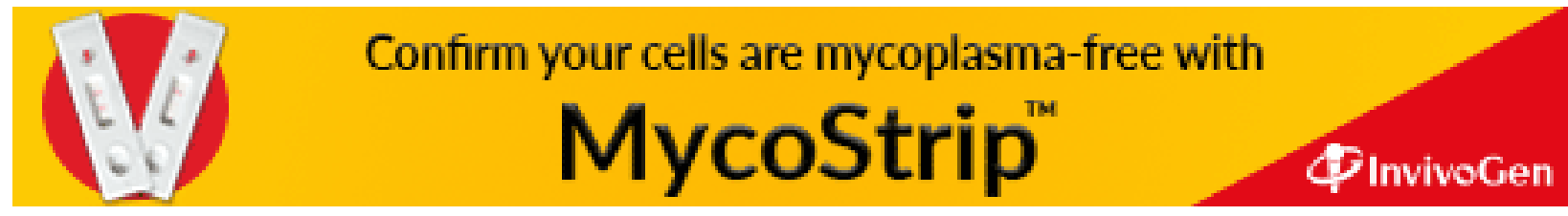

